\title{
A New Modality for Breast Cancer Diagnosis During the COVID-19 Pandemic: A Case Report
}

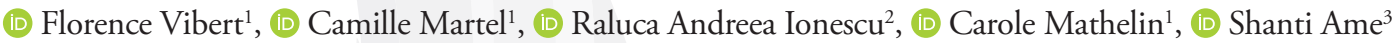 \\ ${ }^{1}$ Department of Surgery, ICANS (Institut de cancérologie Strasbourg Europe), Strasbourg, France \\ ${ }^{2}$ Department of Radiology, Nouvel Hôpital Civil - Hôpitaux Universitaires de Strasbourg, Strasbourg, France \\ ${ }^{3}$ Department of Hemato-oncology, ICANS (Institut de cancérologie Strasbourg Europe), Strasbourg, France
}

\begin{abstract}
Organized screening for breast cancer (BC) was suspended in most countries of the world during the coronavirus disease-2019 (COVID-19) pandemic. Computed tomography (CT) scans of the chest, frequently performed in patients with severe forms of COVID-19, may detect asymptomatic breast abnormalities. A 72-year-old patient, with a severe form of COVID-19 underwent a diagnostic CT scan. This led to the unexpected discovery, at an early stage, of a $12 \mathrm{~mm}$, high grade, Human epidermal growth factor receptor 2 positive BC, with a high proliferation index. After responding to chemotherapy, she was managed with conservative breast surgery with sentinel lymph node biopsy. Delayed management of $\mathrm{BC}$ can be responsible for poor outcomes. Patients with severe forms of COVID-19 are also at risk for developing BC due to common risk factors. Thirty percent of incidental breast lesions discovered on CT scans are undiagnosed BC. Careful study of the mammary glands on CT scan of patients with COVID-19 may allow early diagnosis of a malignant tumor in a high-risk population for $\mathrm{BC}$ and deprived of routine screening mammography.

Keywords: Breast cancer, COVID-19, SARS-CoV-2, screening, chest scan
\end{abstract}

Cite this article as: Vibert F, Martel C, Ionescu RA, Ame S, Mathelin C. A New Modality for Breast Cancer Diagnosis During the COVID-19 Pandemic: A Case Report. Eur J Breast Health 2022; 18(1): 91-93

\section{Key Points}

- Breast cancer screening was suspended in many countries around the world during the COVID-19 pandemic.

- $\quad$ Computed tomography scans of the chest are often performed on the patients with severe COVID-19.

- $30 \%$ of breast lesions incidentally discovered on chest scans are due to cancer.

- Careful study of mammary glands on chest scans in patients infected with SARS-CoV-2 may allow early diagnosis of a malignant tumor in a high-risk population for breast cancer in the absence of routine screening mammography.

\section{Introduction}

Severe acute respiratory syndrome coronavirus 2 (SARS-CoV-2) has been responsible for a pandemic of the condition coronavirus disease-2019 (COVID-19) that has disrupted global health care systems since 2020. Initial publications suggested an increase in the frequency of severe forms of COVID-19 in the elderly or those with significant comorbidities (1). Recommendations were modified for the management of patients with breast cancer (BC). The objective was twofold, not to result in a loss of chance from an oncological perspective, while protecting from a possible COVID-19 infection. The suspension of screening was one of these measures $(2,3)$. Computed tomography (CT) scans, frequently performed in patients infected with SARS-CoV-2, have thus become one of the ways of detecting asymptomatic breast abnormalities.

We report here the case of a patient with high-grade BC diagnosed at an early stage by a CT scan carried out in the context of a SARS-CoV-2 infection, to emphasize the crucial role of chest CT scans.

\section{Case Presentation}

A 72-year-old female patient presented in July 2020 with worsening dyspnea, anosmia and agueusia, after a positive PCR test for SARS-CoV-2. The injected chest CT scan findings with multiple bilateral frosted glass ranges with fibrosis were consistent with a SARS-CoV-2 infection (Figure 1). An incidental $12 \mathrm{~mm}$ breast mass, located in the right breast, was highlighted (Figure 2). Clinical examination of the breast did not 
reveal a mass and there was no suspicious axillary lymph node (LN). The patient was discharged from the infectious disease department after a 10-day hospitalization.

The mammogram carried out a month later revealed a spiculated mass measuring $12 \mathrm{~mm}$, containing microcalcifications that had been absent one year earlier (Figures $3 \mathrm{a}$ and $\mathrm{b}$ ). Breast ultrasound found a hypoechogenic mass, with irregular contours (Figure 4). No suspicious LNs were visualized. A needle core biopsy confirmed a Grade III, infiltrating, ductal carcinoma with highly positive estrogen and progesterone receptors, overexpression of Human epidermal growth factor receptor 2 (HER2), and high proliferation index. Magnetic resonance imagining (MRI) of the breast confirmed these data. Brain and thoracic-abdominal-pelvic CT scans, combined with a bone scan, did not reveal any metastases. The multidisciplinary meeting opted for neoadjuvant chemotherapy and trastuzumab, followed by breast conservation surgery with sentinel LN biopsy, radiotherapy and aromatase inhibitors. The pathological examination of the surgical specimen revealed a complete response.

\section{Discussion and Conclusion}

We report the incidental finding of an early-stage BC during chest imaging carried out as part of the assessment of a SARS-CoV-2 infection in a patient with a normal mammogram one year earlier.

Interruption of $\mathrm{BC}$ screening during the pandemic had been recommended by many international (3) scientific societies. The

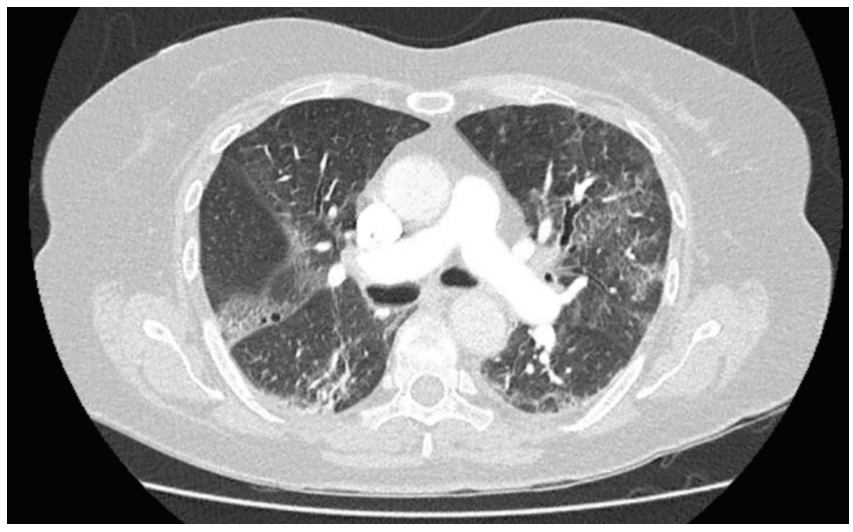

Figure 1. Chest CT scan with multiple, bilateral, frosted glass ranges with fibrosis

\section{CT: Computed tomography}

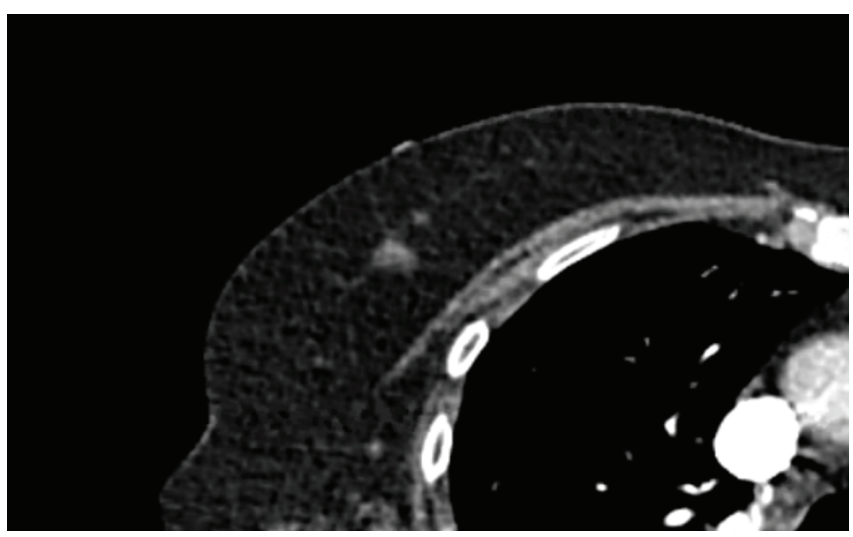

Figure 2. An incidental right breast mass of $12 \mathrm{~mm}$ on chest CT scan rationale for this measure was the potential high risk of SARS-CoV-2 contamination associated with visiting health care centers. Thus, in the absence of a clinical mass, patients did not have access to screening mammograms.

Breast scans present several advantages for diagnosing BC, even in cases of high breast density, without painful manipulation and compression. The diagnostic performance of breast scans is improved by contrast product injection with a reported sensitivity of $90 \%$ [95\% confidence interval (CI): 0.785-0.956] and a specificity of 79\% (95\% CI: 0.709-0.85) (4). In addition, it is more accessible than MRI. In contrast to breast scans, chest CT scans are not dedicated to studying the mammary gland. However, organs present in chest CT scan sectional images, such as the two breasts, can be studied systematically and carefully.

Late diagnosis of $\mathrm{BC}$ and therefore delayed surgery and treatment may be responsible for worsening tumor stages at diagnosis and decreased survival. Bleicher et al. (5) in 2016 and Mateo et al. (6) in 2019 studied 115,790 and 351,087 patients respectively with invasive, non-inflammatory and non-metastatic cancers. They showed a $10 \%$ overall survival decrease per additional month between diagnosis and surgical management (95\% CI: 1.07-1.13; $\mathrm{p}<0.001$; and $95 \%$ CI: $1.08-1.13 ; \mathrm{p}<0.001$, respectively). A large study with more than
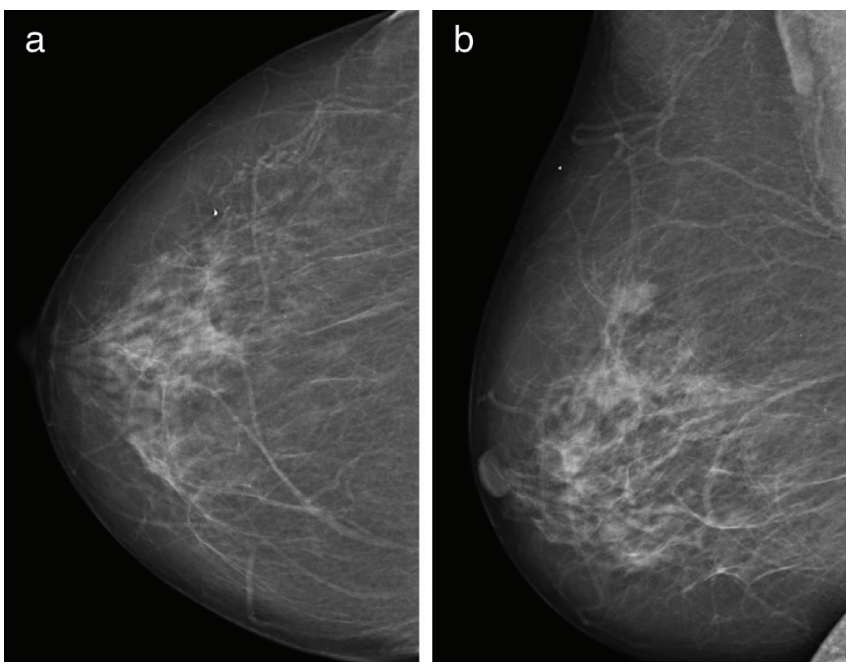

Figure 3. a) The mass was seen on the mammogram (frontal) as a dense, poorly limited, spiculated mass, containing microcalcifications. b) The mass was seen on the mammogram (lateral) as a dense, poorly limited, spiculated mass, containing microcalcifications

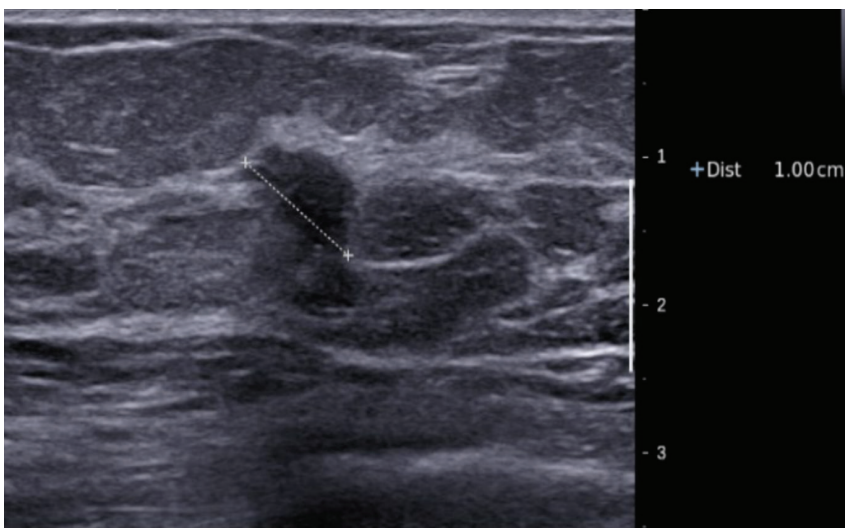

Figure 4. The breast ultrasound found a hypoechogenic mass, with irregular contours and a large vertical axis 
420,000 cases confirmed an excess of mortality [hazard ratio (HR): $1.14,95 \%$ CI: 1.09-1.20] in cases of surgery performed more than 12 weeks after the initial diagnosis, for stage I (HR: 1.19, 95\% CI: 1.11-1.28) and stage II (HR: 1.16, 95\% CI: 1.08-1.25) disease (7). The impact on survival may be related to significant tumor growth, particularly for "aggressive" tumors such as triple negative phenotypes or those overexpressing HER2. A meta-analysis of 2,533,355 patients (8) showed that delayed surgical management of three months resulted in a survival decrease, notably for stage I (HR: 1.27, 95\% CI: 1.161.40 ) and II cancers (HR: 1.13, 95\% CI: 1.02-1.24).

A few months after the onset of the COVID-19 pandemic, the impact of screening cessation on tumor characteristics and survival was studied. Vanni et al. (9) found a statistically different LN invasion rate when comparing 220 patients treated during the pandemic and a similar group of patients treated a year earlier. N2 stages were more frequent $(8 \%$ vs. $2 \%, \mathrm{p}<0.05)$. This locoregional invasion was statistically associated with an extended delay before surgical management [odds ratio (OR): 1.07, 95\% CI: 1.01-1.13, $\mathrm{p}=0.017]$. Thus, according to the predictions of an English group (10), BC mortality could increase from 7.9 to $9.6 \%$ at 5 years due to a delay in diagnosis.

Given screening interruption, the study of the mammary glands on chest CT scans, very often carried out in patients suspected of having COVID-19, appears to be essential. People at risk of serious SARS-CoV-2 infections who require further examination or even hospitalization also present an increased risk for BC. Both diseases have common risk factors, such as advanced age, obesity, and type 2 diabetes $(3,11,12)$. Chest scans performed as part of the diagnosis of COVID-19 infection are generally not accompanied by administration of iodized contrast, except when a pulmonary embolism is suspected, particularly in severe forms (13). However, the injected CT scan is better for exploring the mammary gland. In fact, out of a series of 2,945 patients of all ages who received a chest CT scan, 32 breast lesions were incidentally detected (1.1\%) and 29 of these were identified in injected scans. After further examination, $31 \%$ of the identified lesions were malignant (14). Moyle et al. (15) studied 105,372 scans performed in the general population over a 14-year period. Of the low number of lesions identified $(<1 \%)$, mostly on injected scans $(66 / 78$; $84.6 \%), 28 \%$ were cancerous. The most common cancers identified were invasive carcinomas (14). The lower rate of in situ carcinomas may be explained by the inability to visualize microcalcifications on chest CT scans (15).

Informed Consent: Written informed consent was obtained from the patient for publication of this case report and any accompanying images.

Peer-review: Externally peer-reviewed.

\section{Authorship Contributions}

Surgical and/or Medical Practices: V.F., Ca.M., R.A.I., S.A., C.M.; Concept: V.F., Ca.M., R.A.I., S.A., C.M.; Design: V.F., Ca.M., R.A.I., S.A, C.M.; Data Collection and/or Processing: V.F., Ca.M., R.A.I., C.M.; Analysis and/or Interpretation: V.F., Ca.M., R.A.I., C.M.; Literature Search: V.F., Ca.M., C.M.; Writing: V.F., Ca.M., S.A., C.M.

Conflict of Interest: No conflict of interest was declared by the authors.

Financial Disclosure: This research did not receive any specific grant from funding agencies in the public, commercial, or not-for-profit sectors.

\section{References}

1. Liang W, Guan W, Chen R, Wang W, Li J, Xu K, et al. Cancer patients in SARS-CoV-2 infection: a nationwide analysis in China. Lancet Oncol 2020; 21: 335-337. (PMID: 32066541) [Crossref]

2. Gligorov J, Bachelot T, Pierga JY, Antoine EC, Balleyguier C, Barranger E, et al. [COVID-19 and people followed for breast cancer: French guidelines for clinical practice of Nice-St Paul de Vence, in collaboration with the College Nationale des Gynecologues et Obstetriciens Francais (CNGOF), the Societe d'Imagerie de la Femme (SIFEM), the Societe Francaise de Chirurgie Oncologique (SFCO), the Societe Francaise de Senologie et Pathologie Mammaire (SFSPM) and the French Breast Cancer Intergroup-UNICANCER (UCBG)]. Bull Cancer 2020; 107: 528-537. (PMID: 32278467) [Crossref]

3. Mathelin C, Ame S, Anyanwu S, Avisar E, Mohcen Boubnider W, Breitling $\mathrm{K}$, et al. Breast cancer management during the COVID-19 pandemic: the senologic international society survey. Eur J Breast Health 2021; 17: 188-196. (PMID: 33870120) [Crossref]

4. Uhlig J, Uhlig A, Biggemann L, Fischer U, Lotz J, Wienbeck S. Diagnostic accuracy of cone-beam breast computed tomography: a systematic review and diagnostic meta-analysis. Eur Radiol 2019; 29: 1194-1202. (PMID: 30255249) [Crossref]

5. Bleicher RJ, Ruth K, Sigurdson ER, Beck JR, Ross E, Wong YN, et al. Time to Surgery and Breast Cancer Survival in the United States. JAMA Oncol 2016; 2: 330-339. (PMID: 26659430) [Crossref]

6. Mateo AM, Mazor AM, Obeid E, Daly JM, Sigurdson ER, Handorf EA, et al. Time to Surgery and the Impact of Delay in the Non-Neoadjuvant Setting on Triple-Negative Breast Cancers and Other Phenotypes. Ann Surg Oncol 2020; 27: 1679-1692. (PMID: 31712923) [Crossref]

7. Polverini AC, Nelson RA, Marcinkowski E, Jones VC, Lai L, Mortimer JE, et al. Time to Treatment: Measuring Quality Breast Cancer Care. Ann Surg Oncol 2016; 23: 3392-3402. (PMID: 27503492) [Crossref]

8. Johnson BA, Waddimba AC, Ogola GO, Fleshman JW, Jr., Preskitt JT. A systematic review and meta-analysis of surgery delays and survival in breast, lung and colon cancers: Implication for surgical triage during the COVID-19 pandemic. Am J Surg 2021; 222: 311-318. (PMID: 33317814) [Crossref]

9. Vanni G, Tazzioli G, Pellicciaro M, Materazzo M, Paolo O, Cattadori F, et al. Delay in breast cancer treatments during the first COVID-19 lockdown. A multicentric analysis of 432 patients. Anticancer Res 2020; 40: 7119-7125. (PMID: 33288611) [Crossref]

10. Maringe C, Spicer J, Morris M, Purushotham A, Nolte E, Sullivan R, et al. The impact of the COVID-19 pandemic on cancer deaths due to delays in diagnosis in England, UK: a national, population-based, modelling study. Lancet Oncol 2020; 21: 1023-1034. (PMID: 32702310) [Crossref]

11. Momenimovahed Z, Salehiniya H. Epidemiological characteristics of and risk factors for breast cancer in the world. Breast Cancer (Dove Med Press) 2019; 11: 151-164. (PMID: 31040712) [Crossref]

12. Seiglie J, Platt J, Cromer SJ, Bunda B, Foulkes AS, Bassett IV, et al. Diabetes as a Risk Factor for Poor Early Outcomes in Patients Hospitalized With COVID-19. Diabetes Care 2020; 43: 2938-2944. (PMID: 32847827) [Crossref]

13. Jalaber C, Lapotre T, Morcet-Delattre T, Ribet F, Jouneau S, Lederlin M. Chest CT in COVID-19 pneumonia: A review of current knowledge. Diagn Interv Imaging 2020; 101: 431-437. (PMID: 32571748) [Crossref]

14. Monzawa S, Washio T, Yasuoka R, Mitsuo M, Kadotani Y, Hanioka K. Incidental detection of clinically unexpected breast lesions by computed tomography. Acta Radiol 2013; 54: 374-379. (PMID: 23395815) [Crossref]

15. Moyle P, Sonoda L, Britton P, Sinnatamby R. Incidental breast lesions detected on CT: what is their significance? Br J Radiol 2010; 83: 233-240. (PMID: 19546179) [Crossref] 